\title{
Preclinical episodes of orofacial pain symptoms and their association with healthcare behaviors in the OPPERA prospective cohort study.
}

\author{
Gary D. Slade ${ }^{1,2,3}$, Anne E. Sanders ${ }^{1,2}$, Eric Bair ${ }^{1,4,5}$, Naomi Brownstein ${ }^{5}$, Dawn Dampier 6 , \\ Charles Knott ${ }^{6}$, Roger Fillingim ${ }^{7}$, William O. Maixner ${ }^{1,4}$, Shad Smith ${ }^{1,4}$, Joel Greenspan ${ }^{8,9}$, \\ Ron Dubner $^{9}$, and Richard Ohrbach ${ }^{10}$ \\ ${ }^{1}$ Regional Center for Neurosensory Disorders, University of North Carolina at Chapel Hill, Chapel \\ Hill, NC \\ ${ }^{2}$ Department of Dental Ecology, University of North Carolina at Chapel Hill, Chapel Hill, NC \\ 3 Department of Epidemiology, University of North Carolina at Chapel Hill, Chapel Hill, NC \\ ${ }^{4}$ Department of Endodontics, University of North Carolina at Chapel Hill, Chapel Hill, NC \\ ${ }^{5}$ Department of Biostatistics, University of North Carolina at Chapel Hill, Chapel Hill, NC \\ ${ }^{6}$ Battelle Memorial Institute, Durham, NC \\ 7 Department of Community Dentistry \& Behavioral Science, University of Florida, Gainesville, FL \\ ${ }^{8}$ Brotman Facial Pain Center, University of Maryland-Baltimore, Baltimore, MD \\ 9 Department of Neural and Pain Sciences, University of Maryland-Baltimore, Baltimore, MD \\ 10 Department of Oral Diagnostic Sciences, University at Buffalo, Buffalo, NY
}

\section{Abstract}

\begin{abstract}
The course of preclinical pain symptoms sheds light on the etiology and prognosis of chronic pain.
We aimed to quantify rates of developing initial- and recurrent-symptoms of painful temporomandibular disorder (TMD) and to evaluate associations with health behaviors. In the OPPERA prospective cohort study, 2,719 people aged 18-44 years with lifetime absence of TMD when enrolled completed 25,103 quarterly (three-monthly) questionnaires during amedian 2.3year follow-up period. Questionnaires documented TMD symptom episodes, headache, other body pain, health care attendance and analgesic usage and. Kaplan-Meier methods for clustered data estimated symptom-free survival time. Multivariable models assessed demographic variation in TMD symptom rates and evaluated associations with healthcare and analgesic use. One third of people developed TMD symptoms and for one quarter of symptomatic episodes, pain intensity was severe. Initial TMD symptoms developed at anannual rate of 18.8 episodes per 100 people. The annual rate more than doubled for first-recurrence and doubled again for second-orsubsequent recurrence such that, one year after first recurrence, $71 \%$ of people experienced second
\end{abstract}

(C) 2013 International Association for the Study of Pain. Published by Elsevier B.V. All rights reserved.

Corresponding author: Gary D. Slade, BDSc, DDPH, PhD, Department of Dental Ecology, Koury Oral Health Sciences, Room 4501E, UNC School of Dentistry, 385 South Columbia Street, CB\#7455, USA. Phone: [Int +1] 919-843-0419 gary_slade@ dentistry.unc.edu.

Publisher's Disclaimer: This is a PDF file of an unedited manuscript that has been accepted for publication. As a service to our customers we are providing this early version of the manuscript. The manuscript will undergo copyediting, typesetting, and review of the resulting proof before it is published in its final citable form. Please note that during the production process errors may be discovered which could affect the content, and all legal disclaimers that apply to the journal pertain.

All other authors declare no financial relationships that might represent a possible conflict of interest. 
recurrence. The overall rate increased with age and was greater in African-Americans and lower in Asians relative to Whites. The probability of TMD symptoms was strongly associated with concurrent episodes of headache and body pain and with past episodes of TMD symptoms. Episodes of TMD symptoms, headache and body pain were associated with increases of $\sim 10 \%$ in probability of analgesic usage and healthcare attendance. Yet, even when TMD, headache and body pain occurred concurrently, $27 \%$ of people neither attended healthcare nor used analgesics.

\section{Keywords}

temporomandibular disorder; epidemiology; pain symptoms; prospective cohort study; health behavior

\section{Introduction}

Individuals may experience pain and related symptoms long before a pain condition is diagnosed clinically. These early symptoms may be a preclinical stage in the development of a chronic pain condition that is of prognostic significance. Other symptoms are self-limiting episodes of no consequence. In either scenario, pain symptoms may be singular or recurrent; transient or persistent; mild or severe; and isolated or coexistent with other pain symptoms. Pain intensity may escalate with each episode and their effects might accumulate, such that presence of one preclinical pain symptom contributes to risk of developing a new pain condition[28,30]. Indeed, many people with chronic pain have manifestations that overlap with two or more clinical classifications, although some regional pain conditions have distinctive natural histories. [5] Knowledge about the course of preclinical pain symptoms offers potential for new opportunities to prevent chronic pain.

Longitudinal data regarding pain symptoms are mostly confined to measurement at two points in time. Such studies are informative of risk of onset[19]. For example, in a prospective cohort study of adults aged 18-75 years who were free of orofacial pain on enrollment, $4.6 \%$ developed symptoms after two years[2]. Such studies are also informative of prognosis. Among 14 participating countries in a World Health Organization study of persistent pain syndromes, $49 \%$ of those with a persistent pain condition at enrollment had not recovered 12 months later[12].

However, studies limited to two time points may inadequately measure trends and fail to observe variability in temporal patterns of pain episodes. In a study of orofacial pain that followed adolescents every three months for three years, $10 \%$ of adolescents showed a stepwise pattern of increasing facial pain symptoms over time[9]. The Doetinchem Cohort Study evaluated the 10-year course of low back pain symptoms in an adult population $(n=4,007)$ collecting data on three occasions[29]. Despite stable prevalence over time, the substantial variation in new episodes, recovery, recurrence and long-standing pain led investigators to describe the course of back pain as "a challenge for epidemiological studies" [29](p.998). Similarly, data collected on knee pain at four time points over 12 years showed substantial temporal fluctuation in symptoms[28].

Preclinical pain symptoms are part of the medical "symptom iceberg,"[13] referring to symptoms that are not managed by healthcare professionals. In a 1988 survey of oral and facial pain, only $44 \%$ of those who reported pain symptoms had sought professional attention for their pain[20]. Population-based epidemiological studies are well suited to investigate preclinical pain symptoms which, by definition, are not observed among patients in clinical settings. The four aims of this investigation are to: 
1. Describe demographic variation in the rate of developing TMD pain symptoms prior to clinical diagnosis;

2. Estimate time-to-first-onset and time-to-recurrence of preclinical TMD pain symptom episodes;

3. Evaluate relationships between preclinical TMD pain symptom episodes and two other types of pain symptoms: headache and body pain; and

4. Describe associations between all three pain episodes (preclinical TMD pain symptoms, headache and body pain) and two health behaviors: healthcare attendance and analgesic usage.

\section{Methods}

Results are reported from a prospective cohort study that is part of the OPPERA project (Orofacial Pain: Prospective Evaluation and Risk Assessment)[21]. This paper investigates preclinical symptoms prior to development of examiner-classified temporomandibular disorder (TMD) in OPPERA. "Preclinical" in this study relates to the period "before a disease becomes clinically recognizable"[1]. The OPPERA prospective cohort study continuously monitored symptoms during follow-up of study participants and clinically examined those who reported symptoms. For this study, the preclinical period represented the period between study enrollment and either classification of TMD by OPPERA examiners or the end of data collection, whichever came first. This paper does not investigate predictors of examiner-classified TMD because those findings will be reported in other papers from the OPPERA project.

Methods for the parent OPPERA project are described elsewhere[27]. Methods relevant to this investigation are summarized below and additional details appear in the Supplementary materials.

\section{Enrollment of study participants in the OPPERA prospective cohort study}

Between May 2006 and November 2008, the OPPERA project enrolled an inception cohort of 3,263 people with no history of TMD. Enrollment was at four US study sites: Baltimore, MD; Buffalo, NY; Chapel Hill, NC; and Gainesville, FL. Participants were recruited from the community using advertisements, emails and flyers explaining that healthy volunteers aged 18-44 years were needed for a study of muscle and jaw function. Inclusion criteria were: aged 18-44 years; fluent English language; negative responses to each of 10 questions about significant medical conditions; no history of facial injury or surgery; not receiving orthodontic treatment; not pregnant or nursing. Enrollees also met four symptom-history criteria determined during a screening telephone interview: (i) no orofacial pain in the preceding month and orofacial pain for no more than four days per month in any preceding month; (ii) no more than four headaches per month within the preceding three months; (iii) never diagnosed with TMD; and (iv) no use of a night guard occlusal splint. Two additional selection criteria were established during a clinical examination based on the Research Diagnostic Criteria for TMD[10]: (i) pain reported in the examiner-defined orofacial region for no more than 4 days in the prior 30 days; and (ii) absence of both TMD myalgia and arthralgia. Human subject or institutional review board approvals were received at all four study sites and the data coordination center. All study participants provided written, informed consent before taking part in the study. The OPPERA study is being conducted under the auspices of a Certificate of Confidentiality (NIDCR-06-17) between the National Institutes of Health and Dr William Maixner, Principal Investigator of OPPERA. The Certificate protects the privacy of research subjects. 


\section{Baseline data collection}

At enrollment, telephone interviewers asked about age, gender, race and ethnicity. A demographic questionnaire asked whether or not participants had lived all of the life in the United States. A Comprehensive Pain and Symptom Questionnaire[23] (CPSQ) asked about multiple pain symptoms including "Have you ever had pain in your face, jaw, temple, in front of the ear, or in the ear, not including toothache or ear infection?". Other questions in the CPSQ asked about intensity of headaches during the previous year with response options being "None", "Mostly mild, ranging to moderate", and "Mostly severe, at times moderate". People were also asked if they had experienced "other pains that lasted a whole day or more, or that occurred several times a year due to any cause, and which you still experience".

\section{Follow-up data collection using three-monthly (hereafter "quarterly") health update (QHU) questionnaires}

At three-monthly intervals after enrollment, study participants were asked to complete a quarterly health update (QHU) questionnaire, either online or on paper. In order to capture an approximate three month period of symptoms, the questionnaire was imprinted with a person-specific reference date that was 13 weeks prior to the intended completion date. Questions asked about symptoms experienced in the period since that reference date. Respondents also recorded the date on which they completed each questionnaire. The reporting period is defined here as the number of days between the reference date and the date on which the questionnaire was completed. The intended reporting period was 13 weeks, although its duration varied because some respondents completed the QHU questionnaire earlier or later than intended.

\section{People and QHU questionnaires used in this analysis}

This analysis was limited to 2,719 people who completed 25,103 QHU questionnaires during follow-up (Supplementary Figure 1). Excluded from analysis were five people found to be ineligible for enrollment and 521 people who completed no QHU questionnaires. Individual QHU questionnaires were excluded because the reference period was invalid ( $n=257$ questionnaires with $<30$ days or $>180$ days between the reference date and completion date), because there were missing responses concerning TMD symptoms ( $\mathrm{n}=760$ questionnaires), or because there were missing responses concerning headache or body pain ( $n=544$ questionnaires). This led to the exclusion of 18 people whose follow-up questionnaires were invalid (Supplementary Figure 1). In total, 539 people were excluded due to missing data: 521 who were lost to follow-up, and 18 with invalid questionnaires.

Length of follow-up varied among study participants for three reasons. 1) People were enrolled during a 30-month window creating 30 months of variation when follow-up for all of them was closed for analytic purposes in May, 2011.2) During the follow-up period, 252 symptomatic people were classified as first-onset TMD and hence their subsequent QHU questionnaires were not used for this study of preclinical symptoms. 3) $n=1,534$ people skipped one or more QHU questionnaires, thereby creating a discontinuous period of followup. Most people with discontinuities skipped either one or two QHU questionnaires ( $n=480$ and 318 people, respectively; Supplementary Figure 1), although 335 people skipped five or more QHUs. Hence, follow-up time was computed using the "gap time" method, described below. This provided the denominator to compute symptom rates, therefore providing valid estimates of symptom incidence, even for people who completed fewer questionnaires than intended. 


\section{Classification of pain symptoms and health related behaviors}

Three types of symptom episodes were enumerated for each QHU questionnaire. The exact wording of questions appears in the Supplementary materials, together with findings of testretest reliability in reporting symptom episodes.

i. A TMD symptom episode was defined as a reporting period (defined above) in which orofacial pain for at least 5 consecutive days per month was reported for one or more months. Five days per month was used as the threshold because it exceeded the frequency of TMD symptoms that was permitted at enrollment. This allowed us to classify such TMD symptom episodes as "new" events, which is an important requirement when describing incidence in an inception cohort.-TMD symptom episodes were classified using only these reported symptoms; findings from clinical examinations were not used in this analysis.

ii. An episode of headache was defined as a reporting period in which at least five headaches were reported in the preceding month. Five times per month was used as the threshold because it exceeded the frequency of headaches permitted when enrolling the cohort.

iii. An episode of bodily pain was defined as a reporting period in which ache(s) or pain(s) lasting one day or more were reported in one or more of the following locations: abdomen, arms, back, chest, feet, hands, hips, legs, neck or shoulders. One or more days was used as the threshold for reporting other body symptoms because the underlying focus of this paper, the symptom iceberg, applies to pain conditions of varying duration that can occur in the rest of the body. This threshold provided a more inclusive depiction of what people report. In metaphoric terms, it allows a better estimate of how deep the bottom of the iceberg might extend. For each anatomical location listed in the questionnaire, respondents were asked to check all that applied.

When reporting TMD symptoms, people rated the intensity of the pain using an 11-point numeric rating scale (0: no pain; 10: worst pain imaginable) $[15,16]$. Whether or not pain symptom episodes were reported, people were also asked if they had visited a physician, dentist, chiropractor or any other type of healthcare provider during the reporting period. However, the reason for the visit was not queried. Those who reported having made a visit were asked if the healthcare provider told them that they had "Temporomandibular Muscle and Joint Disorder, TMJD, TMD, or TMJ". Each QHU questionnaire also inquired if respondents were taking pain medications, whether or not these were prescribed by a doctor.

\section{Data analysis}

Except where noted, all data were analyzed using SAS v9.2. Potential biases in loss to follow-up were investigated by comparing baseline characteristics of people who completed one or more QHU questionnaires with people who completed no QHU questionnaires. Chisquare tests for contingency tables were used to evaluate statistical significance of differences in percentages between groups classified according to demographic characteristics and history of pain at baseline.

Dependent variables for Aim 1 were the risk of TMD symptom episodes and the rate of TMD symptom episodes. Risk was defined as the probability of developing a condition, whereas rate was defined as the frequency of a condition within a defined population within a specified time period[19]. Each person was classified as having none, one, two, or three or more episodes, and the percentage of people with one or more episodes represented the risk of TMD symptom episodes. The rate was computed as the number of symptom episodes divided by the sum of reference periods queried in QHU questionnaires. The rate was 
expressed as the number of episodes per 100 people per year. Percentages and rates were computed separately for four demographic groups, classified according to characteristics reported at baseline: 1) three age categories (18-24 years, 25-34 years, 35-44 years); 2) two genders; 3 ) five categories of race/ethnicity (White, Black or African American, Asian, Hispanic, Other or unstated); and 4) three categories of U.S. residence (lifetime US residence, less than lifetime US residence, not stated). Multivariable analysis used a cumulative logistic regression model to evaluate combined effects of demographic characteristics on odds of successively greater numbers of symptom episodes. For this and other multivariable models described below, OPPERA study site was an additional covariate. Demographic variation in the TMD symptom rate was evaluated statistically with Poisson regression using a generalized estimating equation specifying an independent working correlation matrix to allow for clustering of potentially multiple episodes within a person. For each person, the offset variable was the log of the observed follow-up time, producing parameter estimates for indicator variables that represented the log of the rate ratio. One or more indicator variables were created for each demographic category after selecting one category as the referent. Estimates of rate ratios with $95 \%$ confidence intervals (95\% CIs) that excluded the null value of 1.0 were judged to be statistically significant.

For Aim 2, investigation of time-to-onset for initial and recurrent episodes was undertaken with SUDAAN software using its Kaplan-Meier method for survival analysis adapted for clustered data[33]. Survival analysis is necessary for longitudinal studies where data are censored. In this study, right-censoring occurred either when a person developed clinicallydiagnosed TMD, was lost to follow-up, or when data collection stopped in May 2011. TMD diagnosis was included as a censoring criterion because of this paper's focus of preclinical symptoms. Because people could experience more than one event of TMD symptoms, survival times were analyzed using methods appropriate for recurrent event data. Risk intervals were calculated as "gap time" periods and analyzed as restricted risk sets with event-specific baseline hazards[18]. Specifically, time to an initial episode was defined as the observed follow-up time from enrollment up to and including the period in which a first episode of TMD symptoms was reported. If there was no initial episode, the person's observed follow-up time was the censored survival time. For people who developed one episode, a second observation was created in the analytic dataset, representing either time to first recurrence or survival time to censoring. Time to first recurrence was calculated as the observed follow-up time after the first episode up to and including the period in which a second episode of TMD symptoms occurred. Otherwise, the observed follow-up time after the first TMD episode was the censored survival time. The same logic was used to compute time-to-event or time-to-censoring for second recurrence, creating a third observation in the dataset for the same person. This process was repeated for third recurrence, fourth recurrence and so forth, up to the observed maximum of 14 TMD symptom episodes. Survival functions were then estimated for an initial TMD symptoms episode, first recurrence, and second or subsequent recurrence. TMD symptom rates were estimate separately for initial TMD symptoms, first recurrence, and second-or-subsequent recurrence using the same dataset using a generalized estimating equation Poisson regression model with the log of gap-time as the offset variable, as described for Aim 1. For example, the rate of first recurrence was estimated only for people who had initial symptoms, and their gaptime was the interval between the initial symptom and either first recurrence or censoring.

To address Aim 3, a three-way contingency table was created to cross-classify quarterly reporting periods according to presence or absence of TMD symptoms, headache, and body pain, and the counts were plotted in a Venn diagram. The expected number overlapping symptoms was then calculated using a log-linear model for categorical data, specifying no interactions among the three types of symptoms. Additional analysis focused on the proportion of quarterly periods in which TMD symptoms were reported (i.e., risk of TMD 
symptoms) and used the same gap-time dataset used for survival analysis, in which people could be represented by more than one observation. For descriptive purposes, the risk of TMD symptoms was reported in quarterly periods classified according to presence or absence of concurrent headache and body pain during the same quarterly period. Additionally, risk was reported according to presence or absence of symptoms in the person's previous quarterly periods and at the time of enrollment. Tests for differences in risk were evaluated in a multivariable binomial regression model using a generalized estimating equation with an independent working correlation matrix. The model tested for contributions of concurrent and previous pain symptoms to risk of TMD symptoms using quarterly reporting periods as the unit of analysis. People with more than one quarterly reporting period therefore were represented more than once in the dataset, and indicator variables were created to signify whether or not the same person reported symptoms in a previous quarter. An identity link was specified for the model, thereby yielding parameter estimates for predictor variables that represented attributable risk (i.e., risk difference). This permitted modeling of additive effects and additive interactions (i.e., synergy), patterns that were suggested by univariate analysis. Estimates of attributable risk with $95 \%$ confidence intervals (CIs) that excluded the null value of zero were judged to be statistically significant.

Quarterly reporting episodes again were analyzed for Aim 4, focusing on three binary dependent variables: whether or not the person had reported any of: visited a healthcare provider; used analgesic medication; or both behaviors during the reporting period. For descriptive purposes, the percentage of quarters with each health behavior was reported according to presence or absence of TMD symptoms, headache and body pain during the same quarterly period and according to participants' demographic characteristics.

Differences in frequency of health behaviors were evaluated in multivariable binary logistic regression models using a generalized estimating equation with an independent working correlation matrix. Predicted probabilities from the model and their associated 95\% CIs were plotted for combinations of eight pain symptoms and for two contrasting demographic groups selected to illustrate one group with high underlying probabilities of each behavior (40-year-olds, White, females) and another group with low underlying probabilities of each behavior (20-year-old Asian males).

Finally, hot deck multiple imputation[3] was used to evaluate the degree of bias created by loss to follow-up in estimates of symptom rates and rate ratios. The method used five steps. 1) Four characteristics that were associated both with loss to follow-up and with TMD incidence rate were identified: study site (2 categories: Maryland, all other sites), race/ ethnicity (2 categories: Black/African American, all other race/ethnic groups), baseline headache history ( 2 categories: none, mild/severe), and baseline history of body pain ( 2 categories: yes, no). 2) The complete cohort of 3,258 people enrolled at baseline was crossclassified according to those four characteristics, creating sixteen strata; 3) For each of the 521 people lost to follow-up, one person was sampled at random from among the people in the same stratum who provided follow-up data. The method used simple random sampling with replacement. The sampled person's number of symptom episodes and follow-up period were used as the imputed estimates for the person lost to follow-up. 4) The imputed records were added to the records from 2,719 people who had follow-up data, creating a dataset of 3,258 with complete information about symptoms and follow-up period. 5) Steps 3 and 4 were repeated 10 times, with independent random sampling in each replication, and incidence rates and rate ratios were calculated for each of the 10 replicated datasets. The 10 sets of results were combined using the SAS MIANALYZE procedure to generate valid estimates of rates, rate ratios and their $95 \%$ confidence intervals. Hot deck multiple imputation was selected from among other possible methods for multiple imputation because it requires no assumptions about the distribution of variables (which is an important criterion for the follow-up period, which was censored in this study) and it is not affected by 
model misspecification (which can be a problem with regression-based methods of imputation.)[3]

\section{Results}

The percentage of people who provided follow-up data did not vary significantly according to baseline characteristics of age, lifetime US residence, or history of orofacial pain (Supplementary Table 1). However, the percentage was higher in females compared to males, and was lower in African-Americans compared to other racial/ethnic groups. Also, the percentage providing follow-up data was greater among people who reported a history of headache or a history of body pain at enrollment than among people with no such history. The 2,719 people retained in the cohort completed a median of 9 quarterly health update questionnaires (QHUs) (range $=1$ to 22 QHUs) during a median observed follow-up period of 2.3 years (range $=0.12$ to 5.8 years). The median reporting period for the $25,103 \mathrm{QHU}$ questionnaires was 83 days and $90 \%$ of reporting periods were between 73 and 121 days. The median number of QHUs and observed follow-up periods were greater for Whites than for African-Americans, although there were only small differences according to age group, gender and lifetime US residence (Supplementary Table 2).

Two thirds of people (65.5\%) developed no episodes of painful TMD symptoms during follow-up, while $21.0 \%$ developed one episode, $7.7 \%$ developed two episodes, and $5.9 \%$ developed three or more episodes. Only $1.8 \%$ of people experienced five or more TMD symptom episodes, and two thirds (66.0\%) of the 1,675 TMD symptom episodes were reported by only one eighth (13.6\%) of people in the cohort. The percentage of people developing one or more TMD pain symptom episodes did not vary markedly according to the period of follow-up, although among the one quarter of people with fewer than 1.08 years of follow, only $1.18 \%$ developed $\geq 3$ symptom episodes, compared to $7 \%$ or $8 \%$ among people with longer periods of follow-up (Supplementary Figure 2).

In univariate analysis, both the risk of TMD symptom(s) and the rate were greater in successively older age groups, in African-Americans relative to Whites, and in lifetime US residents relative to others (Table 1). The annual TMD symptom episode rate among females (27.9 episodes per 100 females) did not differ from the rate among males (27.7 episodes per 100 males). Multivariable models confirmed that all four demographic characteristics were associated independently with odds of successively greater numbers of incident TMD pain episodes (Supplementary Table 3). Age, race and lifetime US residence, but not gender, were associated independently with the annual incidence rate of TMD pain symptoms.

Initial TMD pain symptoms developed at an annual rate of 18.8 episodes per 100 people (Fig 1A). Following an initial episode, the annual rate of first-recurrence was 52.2 episodes per 100 people while the annual rate of second- or subsequent-recurrence doubled again, to 109.2 episodes per 100 people. There was corresponding variation in estimated symptomfree survival time for initial and recurrent episodes of TMD symptoms (Fig 1B). One year after enrollment, 25\% of people in the cohort developed an initial TMD episode. One year after the initial episode, $49 \%$ of people developed first-recurrence, and one year after recurrence, $71 \%$ of people experienced a second or subsequent recurrence of TMD symptoms. Although not shown in the figures, 1,378 of the 1,675 symptom episodes (82\%) remained preclinical: only 252 episodes led to classification of TMD during clinical examination by OPPERA examiners and only 45 episodes reportedly were diagnosed as TMD by a healthcare provider outside of the study. 
More than two thirds of the 1,675 TMD symptom episodes occurred in conjunction with other pain symptoms, either body pain ( $\mathrm{n}=568$ episodes), headache ( $\mathrm{n}=214$ episodes) or both body pain and headache ( $\mathrm{n}=374$ episodes) (Fig 2). The observed frequency of combined symptoms was greater than would have occurred had the three regional symptoms occurred independently. For example, the combination of all three regional symptoms observed in 374 episodes was more than six times the expected frequency of 58 episodes (Fig 2). Of note, however, was that in $61 \%(n=15,418)$ of the 25,103 reporting periods, none of the three symptoms was reported.

In unadjusted, univariate analysis of the 25,103 QHU questionnaires, the percentage of reporting periods with TMD symptoms varied substantially according to concurrent episodes of headache and bodily pain (Table 2). For example, TMD symptoms were reported in only $3.3 \%$ of quarterly periods when neither headache nor painful body sites were reported, but increased to $27.0 \%$ when there was concurrent headache and concurrent bodily pain at $\geq 2$ sites. TMD symptom occurrence during one quarterly period also varied markedly according to the person's history of TMD symptoms in previous quarterly episodes, from $4.5 \%$ for people with no previous TMD symptom episodes, to $27.0 \%$ for people with two or more previous TMD symptoms episodes. The person's symptom history at baseline was associated with approximately two-fold variation in percentage of painful TMD episodes during follow-up. TMD pain symptoms had a median pain intensity rating of 5 and interquartile range of 3-7. Those values did not vary appreciably according to presence or absence of concurrent or prior pain episodes (Table 2). In multivariable analysis of the data from Table -3 , both concurrent headache and body pain were strongly associated with TMD symptom episodes and they had a small, though statistically significant $(\mathrm{P}=0.008)$ synergistic effect (Table 3). For example, in the absence of concurrent body pain, headache was associated with an increase of $10.5 \%$ in the probability of TMD symptoms, whereas in the presence of two body sites with concurrent pain, headache was associated with an increase of $13.7 \%$ in the probability of TMD symptoms. A person's experience of one previous TMD symptom episode was associated with an additional net increase of 5.2\% in probability of TMD symptoms, while two or more previous TMD symptom episodes were associated with an increase of $16.6 \%$ in TMD symptoms. Headache and body pain at the time of enrollment both were associated with modest increases in probability of TMD symptoms during follow-up. Because this was a multivariable model, the effects of concurrent and prior pain episodes were cumulative. For example, compared to risk of $1.9 \%$ in a period where there were no concurrent or prior episodes of TMD or comorbid pain, the risk of TMD symptoms increased to $35.9 \%$ during a reporting period where both headache $(+10.5 \%)$, one body site $(+1.8 \%)$ and their synergistic effects $(+1.6 \%)$ were experienced by a person who had two previous TMD symptom episodes $(+16.6 \%)$ and who, at baseline, had severe headache $(+2.5 \%)$ and body pain $(+1.0 \%)$.

In univariate analysis, analgesic usage, healthcare attendance for any reason, and either behavior were reported in, respectively, $16.3 \%, 45.4 \%$, and $51.2 \%$ of the quarterly periods (Table 4). Analgesics usage varied from $8.5 \%$ of the quarterly periods where there were no concurrent pain symptoms (i.e. neither body pain nor headache nor TMD symptoms) to $48.5 \%$ of the quarterly periods where all three concurrent pain symptoms were experienced. The corresponding percentages for healthcare attendance were $39.7 \%$ and $55.6 \%$. All three categories of health behavior were reported by much higher percentages of females than males and the likelihood of each behavior tended to be greater in older age groups and in lifetime US residents.

Multivariable models of the data from Table 4 confirmed significant main effects and an interaction effect of body pain and TMD symptoms (Supplementary Table 4). In the model for analgesic usage, there was an additional significant $(\mathrm{P}<0.001)$ interaction between body 
pain and headache. The nature of the interactions is visible in plots of predicted probabilities computed from the multivariable models (Figure 3). Combinations of facial pain and headache were more strongly associated with the probability of each health behavior in the absence of body pain than they were in the presence of body pain. Additional large differences in healthcare behaviors were observed between demographic groups (Fig 3). For a given combination of pain symptoms, there was at least $20 \%$ difference in predicted probability of health behaviors between 20-year-old Asian males and 40-year-old White females - demographic characteristics selected purposefully to represent high- and lowunderlying likelihood of healthcare behaviors.

Baseline characteristics associated both with greater loss to follow-up (Supplementary Table 1) and symptom incidence (Tables 1 and 2) had effects that varied in direction. For example, African-Americans were less likely to be retained in the cohort and they had greater symptom incidence than other races. Meanwhile, people with headache or body pain at baseline were more likely to be retained in the cohort, although they, too, had greater symptom incidence than the referent group of people with no such pain at baseline. When these effects were evaluated simultaneously using multiple imputation, the net effect was a small change in study outcomes. Specifically, the imputed rate of 28.7 symptoms per annum (Supplementary Table 5) was only marginally greater than the unimputed rate of 27.8 from complete-case analysis of people who provided follow-up data (Table 1). Likewise, imputed and unimputed rate ratios for demographic characteristics differed only at the second decimal place (Table 1).

\section{Discussion}

\section{Key findings}

During an average follow-up period of approximately two years, one third of adults in this study developed TMD symptoms that were rarely diagnosed as clinical TMD, and which therefore represented a predominantly preclinical iceberg of symptoms in the community. After developing an initial episode, the TMD symptom rate more than doubled and following first recurrence, people were more likely than not to experience further recurrence within 12 months. Nonetheless, only $6 \%$ of people in the cohort developed three or more episodes of TMD symptoms. The overall rate of TMD symptoms was strongly associated with concurrent episodes of headache and body pain and it varied two-fold according to some demographic characteristics, although not gender. TMD symptom episodes were associated with only a $10 \%$ increase in probability of healthcare attendance and most episodes were tolerated without analgesics.

\section{Temporal patterns of TMD symptoms}

We used three epidemiological measures to summarize distinctive features of symptom onset. The first was TMD symptom risk, which summarized individuals' probability of developing one or more symptom episode at any stage during follow-up. We further distinguished between people who experienced one, two or three-or-more TMD symptom episodes, representing a rudimentary method of clustering individuals according to symptom density. Our finding that multiple episodes developed in only a small proportion of people is consistent with results from a cohort study of adolescents where a more sophisticated method was used to cluster individuals based on temporal trajectories of TMD symptoms[9]. For example, Dunn et al[9] found that most adolescents who developed TMD symptoms experienced relatively short bursts, while only one in ten experienced symptoms through all three years of follow-up. In this study, most people who developed TMD symptoms experienced only one or two episodes, whereas only $6 \%$ of people in the cohort developed three or more episodes. 
The second measure was the rate of TMD symptoms representing the "force of morbidity" [19] because it considers both the number of symptom episodes and rapidity of their onset. When evaluating associations with sociodemographic characteristics, there were only subtle differences between the symptom risk and symptom rate. For example, females had a slightly greater risk than males of developing 1 or 2 TMD symptom episodes, while the symptom rate did not differ. This illustrates that symptom occurrence was equivalent when expressed relative to period of observation, recognizing that women had slightly longer average periods of follow-up than men. Symptom rates were more informative when considered for people with recurrent symptoms. For example, the rate of symptoms more than doubled after an initial episode (Fig 1A). This is an aspect of symptom frequency that cannot be dissected using a measure of symptom risk.

Survival time, the third measure, provided further insight concerning timing of symptoms. To allow for more than one episode per person, we used methods that adapt conventional Kaplan-Meier analysis, permitting repeated observations per person. To our knowledge, survival analysis has not previously been used to describe temporal patterns of TMD symptoms, and the findings (Fig 1B) were noteworthy by showing how quickly recurrence was likely to occur. For example, after experiencing two episodes, $71 \%$ of people experienced further recurrence within 12 months. This represents a useful population benchmark to formulate advice about prognosis for patients who experience repeated episodes of TMD symptoms.

In the analysis of symptom risk, we investigated both individuals and reporting periods as the units of analysis because people and episodes represent distinct populations of interest. The analysis of reporting periods demonstrated greater-than-chance overlap of headache, bodily pain and TMD symptoms (Fig 2), consistent with other studies of widespread pain $[6,17]$ and regional pain $[8,11,32]$. In multivariable analysis of episodes, risk of TMD symptoms was elevated when comorbid conditions occurred contemporaneously or prior to the TMD symptom episode (Table 3). Additionally, risk of TMD symptoms in one episode was strongly associated with prior episodes of TMD symptoms. Concurrent and prior episodes of TMD symptoms, headaches and bodily pain had cumulative effects which, in aggregate, increased risk of TMD symptoms from $2 \%$ in the absence of all such episodes to $36 \%$ in the presence of all such episodes.

\section{Sociodemographic associations}

The overall rate of 27.8 episodes per 100 people, comprising both initial and recurrent episodes, did not vary substantially according to gender, although age- and racial/ethnic group differences were large. The general lack of gender variation is consistent with one other study of preclinical TMD symptoms[31]. In contrast, studies of clinical TMD and chronic TMD symptoms have observed large gender differences. A Swedish study of adolescents reported a three-fold greater risk of examiner-diagnosed TMD in girls compared to boys[22]. In a UK study of chronic orofacial pain symptoms women had more than twice the risk of developing chronic TMD than men[2]. It is possible that gender has little influence on development of preclinical symptoms, but that it affects the transition to clinically-classified TMD or to chronic TMD.

A novel finding was the protective effect of non-lifetime residence in the United States, an effect which persisted after adjustment for race/ethnicity. In addition to foreign-born immigrants to the U.S., non-lifetime residence describes native-born Americans who had lived some time abroad. One explanation of this effect is the healthy migrant phenomenon by which first-generation immigrants are a selectively healthy group[24,25]. It is plausible that US-born people who live abroad temporarily might likewise be relatively healthy, and therefore less prone to pain symptoms. 


\section{The symptom iceberg}

As reported in other population-based studies,[20,26] many of these TMD symptom episodes apparently were not treated, either by healthcare providers or with analgesics. This is despite the finding that one half of TMD symptom episodes were rated as 5 or higher using a 0-10 numeric rating scale, consistent with "moderate" pain seen in clinical settings, and one quarter were rated as 7 or higher, consistent with "severe" clinical pain[4]. Barriers to healthcare probably represent a small part of the explanation; when enrolled, $81 \%$ of people had health insurance, and 57\% had annual household incomes of $\$ 40,000$ or more[27]. Instead, answers probably lie in the salience of pain and its impact on people's daily lives which, together, dictate many aspects of illness behavior[20]. Meanwhile, it was notable that analgesic usage and healthcare attendance were influenced as much by demographic characteristics as they were by pain symptoms. This suggests that demographically-patterned social norms regarding health behaviors also are strong predictors of whether or not pain is treated.

\section{Study limitations}

In prospective cohort studies, loss to follow-up is inevitable and it is likely to cause bias when the degree of loss is differential, i.e. when it occurs unequally in groups defined by predictor or outcome variables. However our analysis showed generally non-differential loss to follow-up (Supplementary Table 2), and when multiple imputation was used to account for loss to follow-up, it produced negligible changes in symptom rates and rate ratios. External validity is limited because study participants were not selected at random from the population. However subjects were recruited from four study sites using multiple community approaches thereby enhancing representation of major U.S. demographic groups.[27] Another restriction was our exclusion of people with serious medical conditions, which probably contributed to underestimation of symptom incidence.

It was surprising that, within a few years, one third of lifetime-asymptomatic adults developed preclinical TMD symptoms in a . This apparently high rate of TMD symptoms raises the question of whether people had simply forgotten past episodes when enrolled. The National Low Back Pain prospective cohort study asked participants to retrospectively recall various aspects of the pain symptoms that they had prospectively reported a decade earlier. When validated against initial reports, the overall kappa statistic of 0.37 indicated only "fair" agreement between initial and recalled pain symptoms[7]. We expect that one feature of the symptom iceberg is that individuals forget prior episodes of a mild or self-limiting nature, especially those that occurred many years earlier.

\section{Clinical and public health significance}

These findings that many TMD symptoms occur as single episodes support a philosophy of very conservative clinical management for patients seeking care for initial onset of painful TMD . Conversely, assigning the label "chronic pain" based only on time since onset of the presenting condition may underestimate a patient's pain history, as earlier episodes may have contributed the presenting complaint. The "iceberg" of TMD symptoms found among one third of people supports the Institute of Medicine's (IOM's) view[14] that the "most prominent feature of pain that qualifies it as a public health problem is its sheer prevalence". While most TMD pain symptom were preclinical, one quarter was of moderate intensity, and one quarter was severe. If the IOM's emphasis on primary prevention was intended to target symptoms of this type, interventions would need to overcome the apparent resistance of people with TMD symptoms to use healthcare. Conversely, among people who can cope adequately with such symptoms, studies investigating their pain management strategies might provide new insights and directions for primary prevention strategies. 


\section{Supplementary Material}

Refer to Web version on PubMed Central for supplementary material.

\section{Acknowledgments}

Funding for this study was provided by NIH/NIDCR U01-DE017018 and R03-DE022595. Drs. Slade is a consultant and equity stock holder in Algynomics Inc., a company providing research services in personalized pain medication and diagnostics. Dr. Maixner is President with equity stock holdings in Algynomics Inc.

\section{References}

1. Dorland's Illustrated Medical Dictionary. W.B. Saunders Co.; Philadelphia, PA: 2012.

2. Aggarwal VR, Macfarlane GJ, Farragher TM, McBeth J. Risk factors for onset of chronic oro-facial pain--results of the North Cheshire oro-facial pain prospective population study. Pain. 2010; 149(2): 354-359. [PubMed: 20304556]

3. Andridge RR, Little RJ. A Review of Hot Deck Imputation for Survey Non-response. Int Stat Rev. 2010; 78(1):40-64. [PubMed: 21743766]

4. Collins SL, Moore RA, McQuay HJ. The visual analogue pain intensity scale: what is moderate pain in millimetres? Pain. 1997; 72(1-2):95-97. [PubMed: 9272792]

5. Croft $P$, Lewis M, Hannaford P. Is all chronic pain the same? A 25-year follow-up study. Pain. 2003; 105(1-2):309-317. [PubMed: 14499449]

6. Dao TT, Reynolds WJ, Tenenbaum HC. Comorbidity between myofascial pain of the masticatory muscles and fibromyalgia. J Orofac Pain. 1997; 11(3):232-241. [PubMed: 9610313]

7. Dawson EG, Kanim LE, Sra P, Dorey FJ, Goldstein TB, Delamarter RB, Sandhu HS. Low back pain recollection versus concurrent accounts: outcomes analysis. Spine (Phila Pa 1976). 2002; 27(9):984-993. discussion 994. [PubMed: 11979175]

8. Diatchenko L, Nackley AG, Slade GD, Fillingim RB, Maixner W. Idiopathic pain disorders-pathways of vulnerability. Pain. 2006; 123(3):226-230. [PubMed: 16777329]

9. Dunn KM, Jordan KP, Mancl L, Drangsholt MT, Le Resche L. Trajectories of pain in adolescents: a prospective cohort study. Pain. 2011; 152(1):66-73. [PubMed: 20971561]

10. Dworkin SF, LeResche L. Research diagnostic criteria for temporomandibular disorders. JCraniomandibDisord. 1992; 6:302-355.

11. Goncalves DA, Camparis CM, Speciali JG, Franco AL, Castanharo SM, Bigal ME. Temporomandibular Disorders are Differentially Associated With Headache Diagnoses: A Controlled Study. ClinJPain. 2011

12. Gureje O, Simon GE, Von Korff M. A cross-national study of the course of persistent pain in primary care. Pain. 2001; 92(1-2):195-200. [PubMed: 11323140]

13. Hannay DR. The 'iceberg' of illness and 'trivial' consultations. J R Coll Gen Pract. 1980; 30(218): 551-554. [PubMed: 7452594]

14. Institute of Medicine. Relieving Pain in America: A Blueprint for Transforming Prevention, Care, Education, and Research. The National Academies Press; Washington, DC: 2011.

15. Jensen MP, Karoly P, Braver S. The measurement of clinical pain intensity: a comparison of six methods. Pain. 1986; 27(1):117-126. [PubMed: 3785962]

16. Jensen MP, Turner JA, Romano JM. What is the maximum number of levels needed in pain intensity measurement? Pain. 1994; 58(3):387-392. [PubMed: 7838588]

17. John MT, Miglioretti DL, LeResche L, Von KM, Critchlow CW. Widespread pain as a risk factor for dysfunctional temporomandibular disorder pain. Pain. 2003; 102(3):257-263. [PubMed: 12670667]

18. Kelly PJ, Lim LL. Survival analysis for recurrent event data: an application to childhood infectious diseases. Stat Med. 2000; 19(1):13-33. [PubMed: 10623910]

19. Last, JM.; Spasoff, RA.; Harris, SS.; Thuriaux, MC. International Epidemiological Association. A dictionary of epidemiology. Oxford University Press; Oxford: New York: 2001. 
20. Locker D. The symptom iceberg in dentistry. Treatment-seeking in relation to oral and facial pain. J Can Dent Assoc. 1988; 54(4):271-274. [PubMed: 3288305]

21. Maixner W, Diatchenko L, Dubner R, Fillingim RB, Greenspan JD, Knott C, Ohrbach R, Weir B, Slade GD. Orofacial Pain Prospective Evaluation and Risk Assessment study--the OPPERA study. J Pain. 2011; 12(11 Suppl):T4-11. e11-12. [PubMed: 22074751]

22. Nilsson IM, List T, Drangsholt M. Incidence and temporal patterns of temporomandibular disorder pain among Swedish adolescents. J Orofac Pain. 2007; 21(2):127-132. [PubMed: 17547124]

23. Ohrbach R, Fillingim RB, Mulkey F, Gonzalez Y, Gordon S, Gremillion H, Lim PF, RibeiroDasilva M, Greenspan JD, Knott C, Maixner W, Slade G. Clinical findings and pain symptoms as potential risk factors for chronic TMD: descriptive data and empirically identified domains from the OPPERA case-control study. J Pain. 2011; 12(11 Suppl):T27-45. [PubMed: 22074750]

24. Razum O. Commentary: of salmon and time travellers--musing on the mystery of migrant mortality. Int J Epidemiol. 2006; 35(4):919-921. [PubMed: 16847016]

25. Razum O, Zeeb H, Rohrmann S. The 'healthy migrant effect'--not merely a fallacy of inaccurate denominator figures. Int J Epidemiol. 2000; 29(1):191-192. [PubMed: 10750623]

26. Schiffman EL, Fricton JR, Haley DP, Shapiro BL. The prevalence and treatment needs of subjects with temporomandibular disorders. J Am Dent Assoc. 1990; 120(3):295-303. [PubMed: 2312948]

27. Slade GD, Bair E, By K, Mulkey F, Baraian C, Rothwell R, Reynolds M, Miller V, Gonzalez Y, Gordon S, Ribeiro-Dasilva M, Lim PF, Greenspan JD, Dubner R, Fillingim RB, Diatchenko L, Maixner W, Dampier D, Knott C, Ohrbach R. Study methods, recruitment, sociodemographic findings, and demographic representativeness in the OPPERA study. J Pain. 2011; 12(11 Suppl):T12-26. [PubMed: 22074749]

28. Soni A, Kiran A, Hart DJ, Leyland KM, Goulston L, Cooper C, Javaid MK, Spector TD, Arden NK. Prevalence of reported knee pain over twelve years in a community-based cohort. Arthritis Rheum. 2012; 64(4):1145-1152. [PubMed: 22180258]

29. van Oostrom SH, Monique Verschuren WM, de Vet HC, Picavet HS. Ten year course of low back pain in an adult population-based cohort--the Doetinchem cohort study. Eur J Pain. 2011; 15(9): 993-998. [PubMed: 21429779]

30. Von Korff M, Le Resche L, Dworkin SF. First onset of common pain symptoms: a prospective study of depression as a risk factor. Pain. 1993; 55(2):251-258. [PubMed: 8309712]

31. Widmalm SE, Christiansen RL, Gunn SM. Race and gender as TMD risk factors in children. Cranio. 1995; 13(3):163-166. [PubMed: 8949855]

32. Wiesinger B, Malker H, Englund E, Wanman A. Does a dose-response relation exist between spinal pain and temporomandibular disorders? BMCMusculoskeletDisord. 2009; 10:28. 28.

33. Williams RL. Product-limit survival functions with correlated survival times. Lifetime Data Anal. 1995; 1(2):171-186. [PubMed: 9385099] 


\section{Two sentence summary}

One-in-three adults developed TMD symptoms over 2.3 years. Comorbid pain was common and recurrence was rapid, yet many did not seek healthcare or use analgesics. 

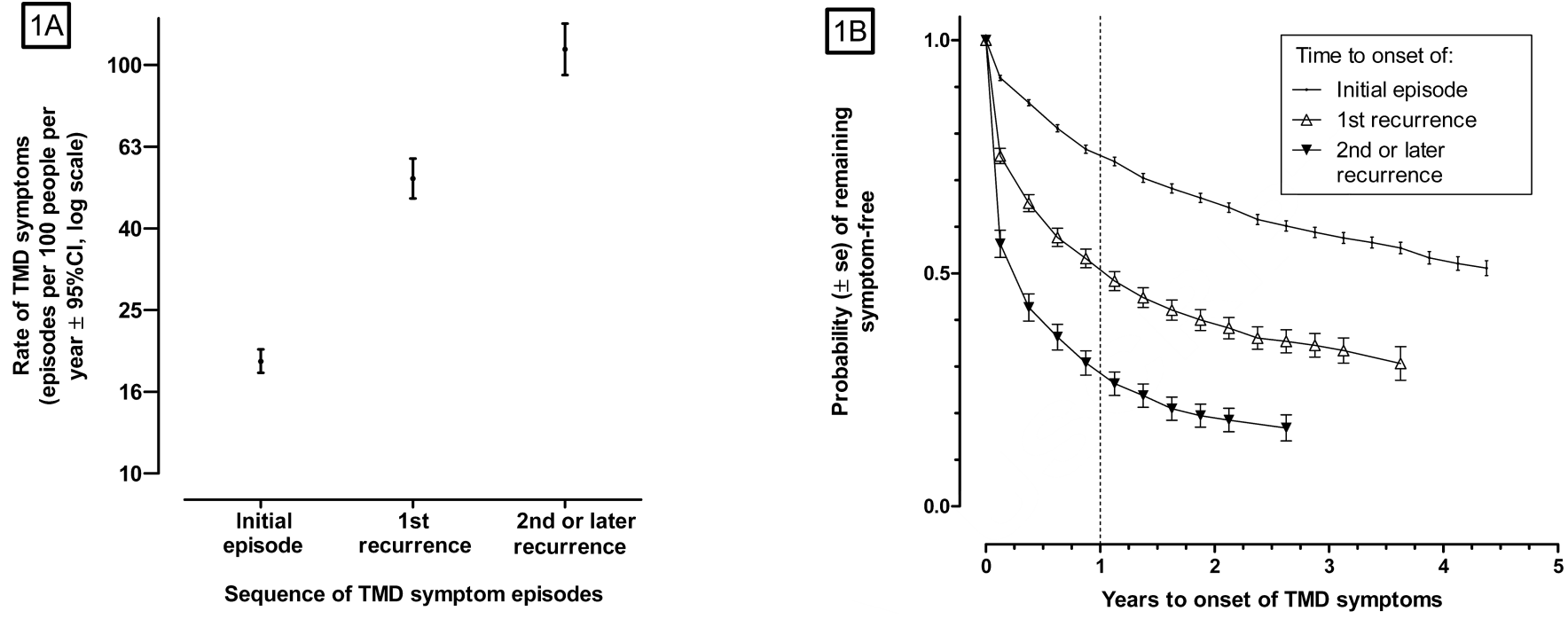

Figure 1. Symptom episode rates and symptom-free survival curves for an initial episode, $1^{\text {st }}$ recurrence and $2^{\text {nd }}$ or subsequent recurrence of TMD symptoms: OPPERA prospective cohort study 2006-2011

The rate of TMD symptom episodes (Fig 1A) was calculated using a Poisson regression generalized estimating equation model that accounted for the correlated data structure created by potentially-multiple episodes of TMD pain symptoms per person. The numerator for the rate was the number of painful TMD symptom episodes, and the denominator was the sum of observed reference periods (in person-years) captured by quarterly (threemonthly) questionnaires. The model adjusted for OPPERA study site. Observed episode rates per 100 people per year were 18.8 for first onset, 52.7 for 1 st recurrence, and 109.2 for 2nd or later recurrence. Symptom-free survival curves (Fig 1B) are from Kaplan-Meier estimates calculated with SUDAAN software that make adjustment for the correlated data structure created for repeated symptom episodes within individuals. For both methods, time to onset for the initial episode is defined as observed person-years from enrollment to development of a first-episode of TMD symptoms. Time to onset for a 1st recurrence is defined as observed person-years from the development of the initial episode to the development of a second episode. Time to onset of a second recurrence is defined as observed person-years from the development of the second episode to the development of a third episode. Similar logic was used for other episodes of recurrence. Estimated probabilities of symptoms after one year were 0.25 for an initial episode, 0.49 for $1 \mathrm{st}$ recurrence and 0.71 for 2 nd or later recurrence. Data for both figures are from 25,103 quarterly questionnaires completed by 2,719 people over a median 2.3 year follow-up period in the OPPERA prospective cohort study. 


\section{$\mathrm{n}=25,103$ questionnaires}

No pain episodes $(n=15,418)$

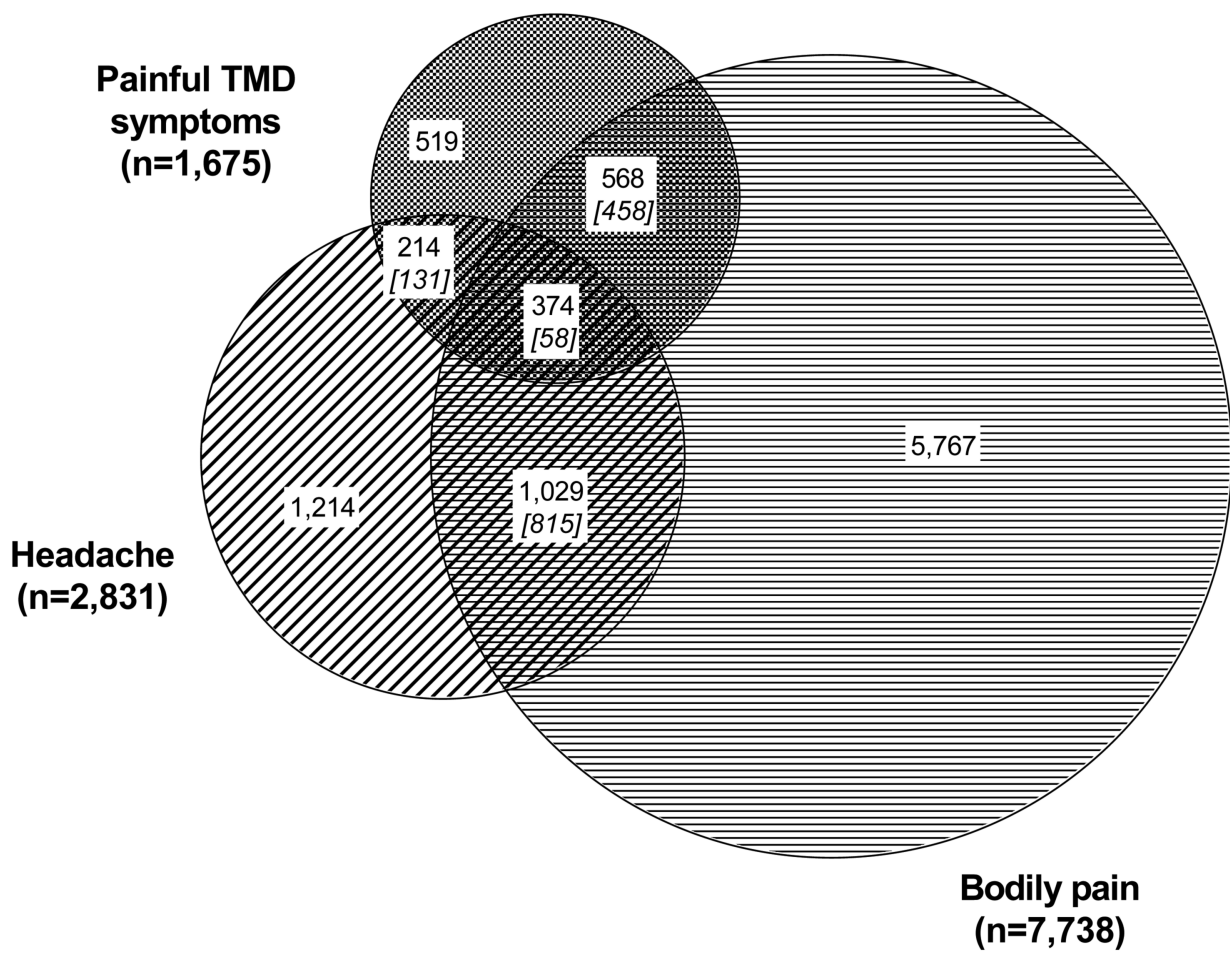

Figure 2. Co-occurrence of regional pain symptoms reported in quarterly reporting periods: OPPERA prospective cohort study 2006-2011

Data are from 25,103 quarterly (three-monthly) questionnaires completed by 2,719 people over a median 2.3-year follow-up period in the OPPERA prospective cohort study.

Questionnaires asked about three types of pain symptoms during the preceding three-month (average) period. Painful TMD symptoms were reports of TMD-like pain lasting $\geq 5$ days per month for $\geq 1$ month of the reporting period. Headaches are reports of $\geq 5$ headaches within the most recent month of the reporting period. Body pain was a report of pain in $\geq 1$ of 10 body locations lasting for $\geq 1$ days in the same reporting period. Numbers in white boxes represent the observed number of episodes. Italicized numbers in square brackets are the expected number of jointly-occurring pain episodes that would have occurred if each type of pain episode occurred independently of the other pain episodes. 

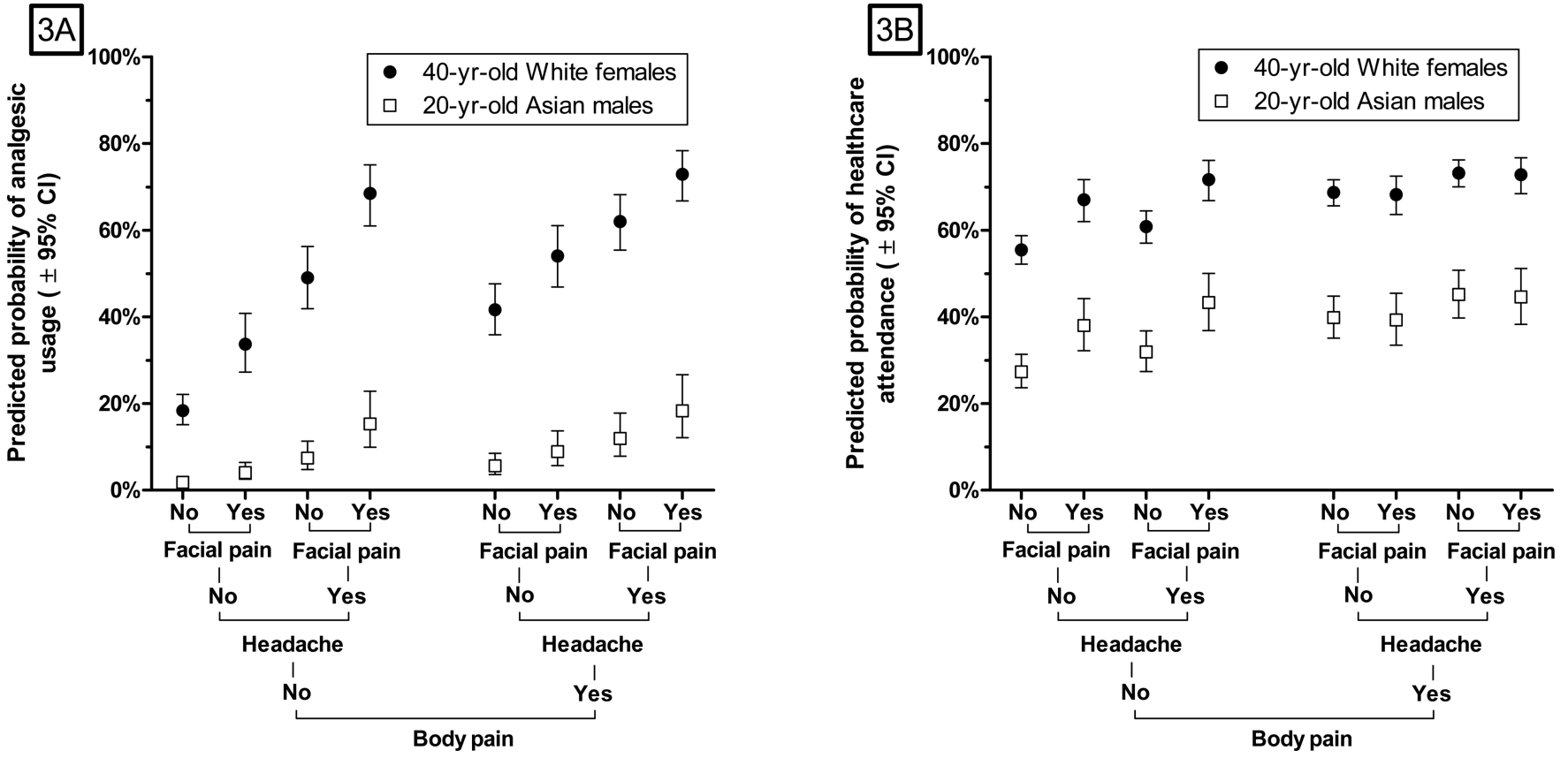

Figure 3. Association between three types of pain symptoms and two health behaviors: OPPERA prospective cohort study 2006-2011

Figure 3: Predicted probabilities of: analgesic usage (Figure 3A) and of healthcare attendance (Figure 3B) during quarterly periods of follow-up in the OPPERA prospective cohort study. Predicted probabilities are from binary logistic regression generalized estimation equation models, shown in Supplementary Table 4. The models estimate probability of each health-related behavior during the same quarter in which symptoms of orofacial pain, headache and bodily pain were reported. Models adjust for OPPERA study site, age (continuous variable), gender (two categories), race/ethnicity (5 categories) and lifetime US residence (three categories). Predicted probabilities and their $95 \%$ confidence intervals are shown for two contrasting demographic groups, selected to illustrate one group with high underlying probabilities of each behavior [ 40-year-olds, White, females] and another group with low underlying probabilities of each behavior [ $\square$ 20-year-old Asian males]. Data are from 25,103 quarterly (three-monthly) questionnaires completed by 2,719 people over a median 2.3 year follow-up period in the OPPERA prospective cohort study. 


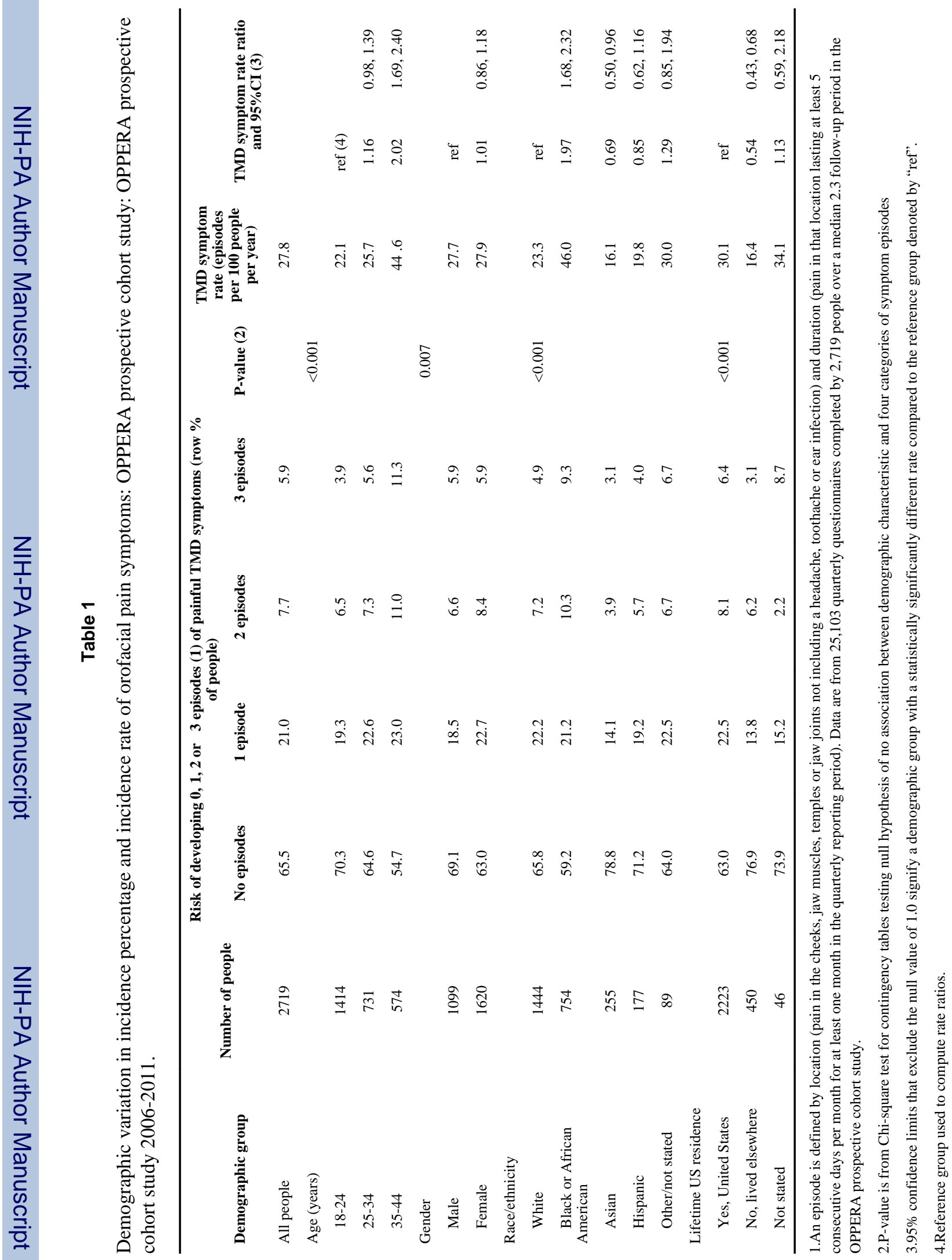

Pain. Author manuscript; available in PMC 2014 May 01. 
Table 2

Univariate associations of concurrent and previous symptom episodes and symptoms at enrollment with probability of TMD symptoms and pain intensity rating: OPPERA prospective cohort study 2006-2011.

\begin{tabular}{|c|c|c|c|c|}
\hline & & Number of questionnaires & $\begin{array}{c}\text { n (\%) of TMD symptom } \\
\text { episodes }\end{array}$ & $\begin{array}{l}\text { TMD pain intensity (1) } \\
\text { (median [IQR]) }\end{array}$ \\
\hline \multicolumn{2}{|c|}{ All questionnaires } & 25103 & $1675(6.7)$ & $5[3,7]$ \\
\hline \multicolumn{5}{|c|}{ Concurrent pain episodes } \\
\hline Headache & Painful body sites & & & \\
\hline \multirow[t]{3}{*}{ No } & 0 & 15937 & $519(3.3)$ & $5[3,6]$ \\
\hline & 1 & 3161 & $231(7.3)$ & $5[3,6]$ \\
\hline & 22 & 3174 & $337(11.0)$ & $5[4,7]$ \\
\hline \multirow[t]{3}{*}{ Yes } & 0 & 1428 & $214(15.0)$ & $5[4,7]$ \\
\hline & 1 & 568 & $146(26.0)$ & $6[5,7]$ \\
\hline & $\geq 2$ & 835 & $228(27.0)$ & $6[4,7]$ \\
\hline \multicolumn{5}{|c|}{ Prior quarterly episodes } \\
\hline \multicolumn{5}{|c|}{ TMD symptom episodes } \\
\hline 0 & & 20822 & $939(4.5)$ & $5[3,7]$ \\
\hline 1 & & 2919 & $369(13.0)$ & $5[4,7]$ \\
\hline$\geq 2$ & & 1362 & $367(27.0)$ & $5[4,7]$ \\
\hline \multicolumn{5}{|c|}{ Headache episodes } \\
\hline 0 & & 18358 & $940(5.1)$ & $5[3,7]$ \\
\hline 1 & & 3392 & $347(10.0)$ & $5[4,7]$ \\
\hline$\geq 2$ & & 3353 & $388(12.0)$ & $6[4,7]$ \\
\hline \multicolumn{5}{|c|}{ Body pain episodes } \\
\hline 0 & & 19960 & $1133(5.7)$ & $5[3,7]$ \\
\hline 1 & & 2929 & $269(9.2)$ & $5[4,7]$ \\
\hline$\geq 2$ & & 2214 & $273(12.0)$ & $5[4,7]$ \\
\hline \multicolumn{5}{|c|}{ Baseline symptom history } \\
\hline \multirow[t]{2}{*}{ Facial pain } & No & 21353 & $1289(6.0)$ & $5[4,7]$ \\
\hline & Yes & 3750 & $386(10.0)$ & $5[3,7]$ \\
\hline \multirow[t]{2}{*}{ Body pain } & No & 22263 & $1379(6.2)$ & $5[4,7]$ \\
\hline & Yes & 2840 & $296(10.0)$ & $5[3,7]$ \\
\hline \multirow[t]{3}{*}{ Headache } & None & 7096 & $372(5.2)$ & $5[4,7]$ \\
\hline & Mild & 16208 & $1073(6.6)$ & $5[3,7]$ \\
\hline & Severe & 1799 & $230(13.0)$ & $6[5,7]$ \\
\hline
\end{tabular}

(1) From 0-10 numerical rating scale of pain intensity reporting during quarterly period in which TMD symptom episodes were experienced. IQR = interquartile range. Data are from 25,103 quarterly (three-monthly) questionnaires completed by 2,719 people over a median 2.3 year follow-up period in the OPPERA prospective cohort study. 
Table 3

Multivariable associations of concurrent and previous symptom episodes and symptoms at enrollment with risk of TMD symptoms: OPPERA prospective cohort study 2006-2011.

\begin{tabular}{|c|c|c|c|}
\hline \multirow{2}{*}{$\begin{array}{l}\text { Risk predictor } \\
\text { Reference probability(3) }\end{array}$} & \multicolumn{2}{|c|}{ TMD symptom probability (\%) and $95 \%$ CL (1) } & \multirow{2}{*}{$\frac{\text { P-value (2) }}{<0.001}$} \\
\hline & 1.9 & $1.4,2.5$ & \\
\hline \multicolumn{4}{|c|}{ Additive probability (4) associated with concurrent symptom episodes } \\
\hline Headache (ref=None) & 10.5 & $8.7,12.3$ & $<0.001$ \\
\hline Number of painful body sites & 1.8 & $1.5,2.2$ & $<0.001$ \\
\hline Synergy: headache and body pain & 1.6 & $0.4,2.9$ & 0.008 \\
\hline \multicolumn{4}{|c|}{ Additive probability associated with previous TMD symptom episodes } \\
\hline 1 episode (ref=None) & 5.2 & 4.06 .5 & $<0.0001$ \\
\hline$\geq 2$ episodes (ref=None) & 16.6 & 13.120 .2 & $<0.0001$ \\
\hline \multicolumn{4}{|c|}{ Additive probability associated with baseline pain history } \\
\hline Mild headache (ref=None) & 0.5 & $0.1,0.9$ & 0.030 \\
\hline Severe headache $(\mathrm{ref}=$ None $)$ & 2.5 & $1.0,3.9$ & 0.001 \\
\hline Body pain (ref=None) & 1.0 & $0.0,2.0$ & 0.044 \\
\hline
\end{tabular}

1)Except as noted for footnote 3 and 4, estimates represent attributable risk (i.e. additive risk difference) of TMD symptoms associated with each risk predictor. Probabilities were estimated using multivariable log-binomial, generalized estimating equation model, specifying an identity link function to model attributable risk of TMD symptoms associated concurrent and past symptoms. The model adjusts for correlation created by multiple observations per person. The multivariable model contained all the concurrent and past symptoms shown in the table, as well as indicator variables for OPPERA study site. Data were 25,103 quarterly (three-monthly) questionnaires completed by 2,719 people over a median 2.3-year follow-up period in the OPPERA prospective cohort study. $95 \% \mathrm{CL}=95 \%$ confidence limits. Confidence limits that exclude the null value of zero show statistically significant additive effects of the relevant symptom on probability of TMD symptoms.

2)P-values are from score statistics from generalized estimating equation analysis of multivariable, log-binomial regression models. The P-value tests the null hypothesis that the estimated additive or synergistic effect equals zero.

3)Estimated probability of TMD symptom episodes per quarterly reporting period in people who had none of the headache or body pain symptoms reported for the same quarterly period.

4)The additional probability of TMD pain symptom episodes due to synergy (additive interaction) between concurrent headache and number of painful body sites. 


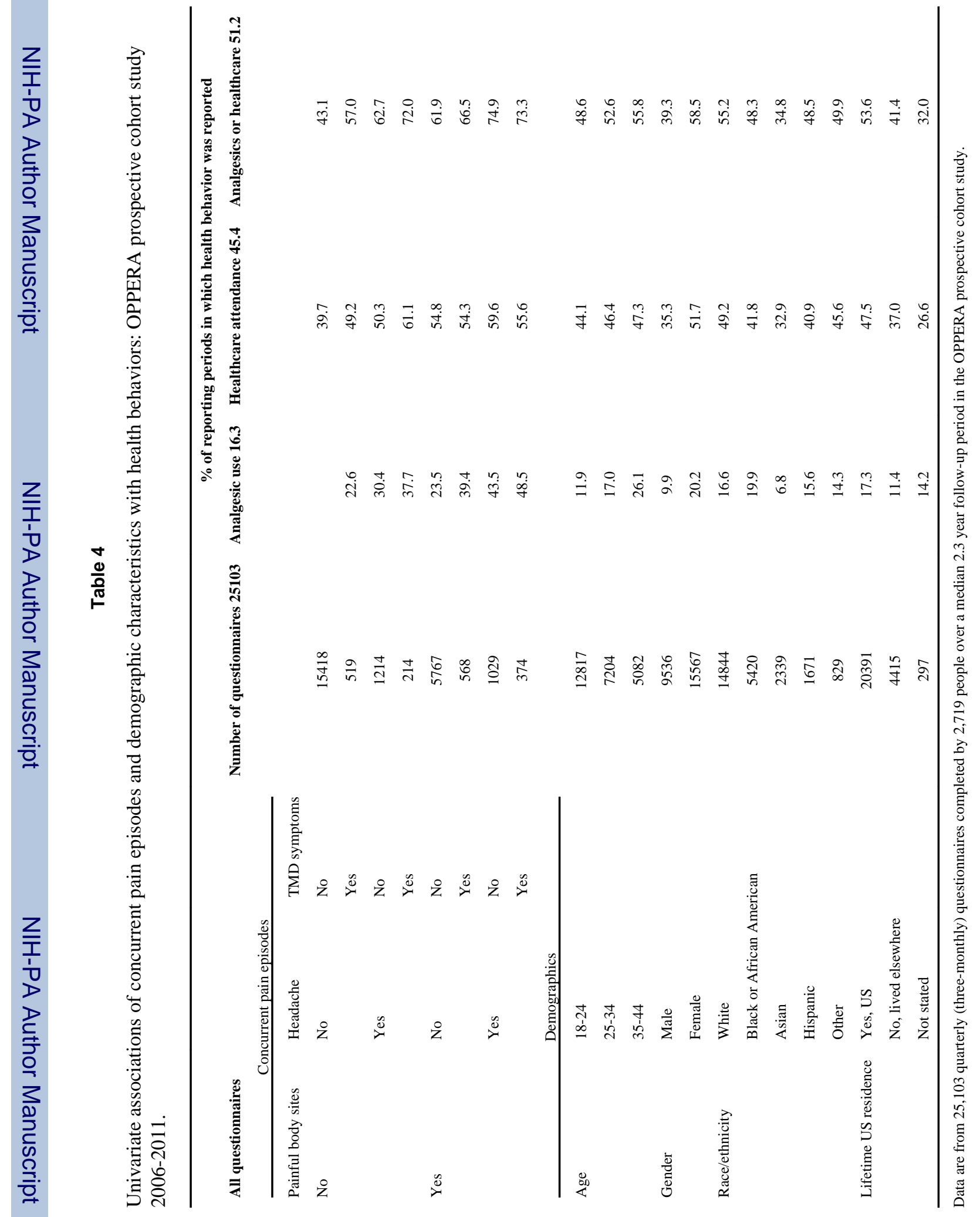

